paediatric unit in a non-tertiary centre over a 3 year period, highlighting the challenges presented.

7 patients had a total of 12 in-patient stays on a general paediatric ward. Length of stay ranged from 6 to 84 days, mean 44 days, median 55 days. Initial presentations were all unscheduled episodes of care. Age at initial presentation ranged from 10 to 14 years. Restrictive type eating disorder was the working diagnosis initial presentation in 5 of 7 cases. All were managed according to the Junior MARSIPAN Guidelines (Royal College of Psychiatrists). In the 2 youngest patients, both 10 years, the diagnosis of an eating disorder was made after medical investigation of weight loss. 5 of 7 children required nasogastric re-feeding during their first admission. 4 of 7 required transfer to a tertiary unit specializing in eating disorder management. Only one of these four was successfully discharged home by 6 months, the others have required repeat admissions over 3 years. Diagnosis in all 7 was restrictive eating pattern, non-bulimic.

Medical complications included profound bradycardia and hypotension at presentation in $7 / 7$ and re-feeding syndrome in 1/7. 2/7 manifested extreme behavioural challenge on a general paediatric ward. 1/7 self-harmed. 24 hour supervision including meal support was required by Health Care Assistants in 5/7. No adolescent/child segregation could be provided. There are no nursing staff with training in managing the child with an eating disorder on our ward. Paediatric dietetic support was available but no other specialist treatment was possible.

This cohort is small in number relative to the overall admissions to our unit. Prolonged and repeat admissions have significant implications for the limited budget of a small hospital. Total length of stay of 489 days cost 400,000 euro for basic care alone. This unscheduled care group also represents a considerable work load for general paediatricians.

Best practice management of Anorexia Nervosa prompts the need for a multi-disciplinary approach from a paediatrician, psychiatrist, psychologist, and speciality trained dietician and nursing staff. The increasing incidence and prevalence of eating disorders among older children and adolescents is an increasing burden of care for general paediatric units in Ireland compounded by an under-resourced community Child and Adolescent Mental Health service.

\section{P406 TRANSGENDER YOUTH: WHAT PAEDIATRICIANS NEED TO KNOW}

${ }^{1}$ Aileen Murtagh*, ${ }^{2}$ Louisa Kelly. ${ }^{1}$ St Patricks Mental Health Services, Dublin, Ireland; ${ }^{2}$ Our Lady's Children's Hospital, Crumlin, Dublin, Ireland

10.1136/archdischild-2019-epa.752

An increase in referral rates of transgender youth to services has been observed on an international level (Chen et al 2016, Fuss et al 2015). There is a paucity of knowledge vis-a-vis paediatrician's experience and knowledge of caring for transgender youth. General paediatricians knowledge is limited, with training on provision of routine care, supporting transgender patients and their families and co-ordination of medical management recommended (Shires et al 2017). Paediatric endocrinologists may be involved in prescription of hormonal therapy in transgender youth, in accordance with the updated Clinical Practice Guideline by the Endocrine Society in 2017.
Gender identity and expression is best conceptualized as a spectrum, and not simply a binary concept. The term transgender can be considered an umbrella term, encompassing the broad spectrum of individuals who transiently or persistently identify with a gender different from their assigned sex at birth. An inversion of the sex ratio of referred adolescents has been observed, with a number of clinics reporting more youth assigned female at birth referred in recent years than youth assigned male at birth (Aitken et al 2015; Kaltiala-Heino et al 2015).

This presentation will include an overview for paediatricans. The gender spectrum, social and medical transitioning, common co-morbidities, current evidence-base \& guidelines and practical management of gender variant youth in the paediatric setting will be covered.

Medical, neurodevelopmental and psychiatric co-morbidities will be highlighted. Gender Dysphoria and comorbidity with medical disorders, such as Kleinfelter Syndrome, and neurodevelopmental disorders, such as Autistic Spectrum Disorders (ASD) and Attention Deficit Hyperactivity Disorder (ADHD), will be explored. The current literature reports high co-occurance rates of Gender Dysphoria and ASD (Van Der Miesen et al 2016, Janssen et al 2016, Shumer et al 2016) and is the focus of much attention. Research in recent years highlights elevated rates of co-morbid mental health difficulties, selfharming behavior and suicidality among transgender youth (Chen et al 2016, Holt et al 2016, Kaltiala-Heino et al 2015, Olson et al 2015, Reisner et al 2015).

\section{P407 CONVERSION DISORDER: DON'T BE MISTAKEN, DON'T BE MISLED}

Evanne O'Halloran*, Aoife Branagan, Niofa Canty, O'Neill Michael B, Hilary Stokes. Department of Paediatrics, Mayo University Hospital, Castlebar, Co. Mayo, Ireland

\subsection{6/archdischild-2019-epa.753}

Conversion disorder is the loss or alteration of voluntary motor, or sensory function in the absence of identifiable pathology. ${ }^{1}$ It is most prevalent in the $10-15$ year old age group, female: male ratio, 2:1.

We present 5 cases: all female, 11-14 years, presenting with vague symptomatology and normal investigations. 3/5 had identifiable psychological stressors. They had a combined total of 15 presentations, 9 admission episodes, 40 in-patient bed days and 20 radiological investigations.

$1 / 5$ was diagnosed with a treatable neurological condition.

Case 1: EO, 11 years old, repeated presentations with generalised weakness, dizziness, headache and arthralgia. Patient's father had died tragically one year prior. Mother has fibromyalgia and functional neurological disorder.

Case 2: RM, 14 years old, multiple episodes of collapse, with apparent unconsciousness (up to 40 minutes) with repeated presentations to hospital. Postulated aetiologies included neurocardiac syncope and Postural Orthostatic Tachycardia Syndrome. There followed exclusion from school and multiple medical opinions.

Case 3: KO, 14 years old, presented following recurrent pre-syncope, collapse, and shaking episodes. Previously investigated for recurrent vomiting, with no aetiology found. Psychological stressors included disabled sibling, significant mental health disorders, both parents. 
Case 4: AO, 14 years old, recurring presentations to the emergency department with stuporous state, collapse, weakness, dizziness, headache, nausea. Perceived pressure to achieve academic excellence seems the psychological stressor. There followed exclusion from school for some months, on health and safety grounds.

Case 5: CM, 13 years old, presented with general malaise, arthralgia, headache and dizziness, with ataxic gait. Difficult transition to secondary school, and involvement in road collision 6 months previous noted. Repeated presentations with worsening symptoms. Objective difficulty eliciting lower limb deep tendon reflexes. Diagnostic lumbar puncture, $3^{\text {rd }}$ admission, demonstrated elevated CSF protein. Subsequent development of symmetrical lower limb paraesthesia. Confirmed diagnosis, Guillain Barre syndrome, responsive to immunoglobulin therapy.

Conversion disorder is a diagnosis of exclusion and careful systematic approach should be applied, ensuring all organic causes of symptoms have being explored, before attributing symptoms to a psychogenic disorder. ${ }^{2,3}$

\section{REFERENCES}

1. WHO. The ICD-10 classification of mental behaviour disorders 1992. Geneva: World Health Organisation.

2. Evans JR. Pediatric Pain Letter June 2015;17:2.

3. Stone J. The bare essentials: Functional symptoms in neurology. Pract Neurology 2009:9:179-89.

\section{P408 CAMHS PATIENTS ADMISSION IN CAVAN GENERAL HOSPITAL OVER 5 YEARS (2013 TILL 2017)}

Sana Marium Ali*, Alan Finan, Caroline A Hearne.

10.1136/archdischild-2019-epa.754

Hse, Cavan, Ireland

Background 1 in 10 children and adolescent suffer from mental illness associated with considerable distress, this audit is done to focus on CAMHS inpatient admissions having suicidal ideation, eating disorder (anorexia nervosa/bulimia nervosa), anxiety disorder prevailing in Ireland in Cavan Monaghan community over 5 years and lack of dedicated pediatric liaison psychiatric team nationally that should be considered to be more active due to increasing ratio of CAMHS inpatient admissions in pediatric wards in local hospitals.

Study by National suicide Research Foundation shows deliberate self harm rose by $22 \%$ over 10 yrs to the end of 2016 , more pronounced in female of age 10 to $14 \mathrm{yrs}, 70$ children of school going age died by suicide in 2017

Aims Over many years pediatric services has supported Cavan and Monaghan community based CAMHS services in the inpatient care of patients with various mental illness, this audit aims to assess the resources given to the care of these inpatients and the changes of workload over recent years

Standards National model of care in pediatric health care services in Ireland.

Survey of inpatient admission of children and adolescent with mental health RCPSYCH 2015

Quality Improvement network for multi agency CAMHS (QINMAC)

Difficulties of high risk patient in pediatric ward with reported self harm incidents on wards, the need of restraint, security staff and properly trained staff and police
Long waiting lists should be eliminated, should provide supportive and comfortable environment to patients with mental illness by trained staff.

Methodology Literature review of most recent Irish or International consensus guidelines on inpatient care of CAMHS patient.

Audit of number of patients under CAMHS admitted over 5 yrs (2013 till 2017)

Pull charts Data collection :

Name

D.O.B

MRN Age at admission

Date of admission

Diagnosis Length of inpatient stay

Result of 2018 audit.

Recommendation for improvement

Results 125 inpatient admission with the diagnosis of suicidal ideation, eating and anxiety disorder (jan 2013 till dec 2017)

49 female $(68 \%)$ and 23 male (32\%)

Average age 14 yrs.

Average Length of stay 6 days, median 3 days

Over 5 yrs 54\% diagnosed suicidal ideation, $24 \%$ eating disorder, $15.16 \%$ anxiety disorder.

Conclusion 2017 peak of new admissions of all the three mental illness.

Female predominance in suicidal ideation and eating disorder

Male predominance in anxiety disorder

Recommendation To assess number of beds need for future.

Meet CAMHS for future service planning.

Re audit.

safeguarding .

Additional staff training.

\section{P409 CHILDREN IN DISTRESS - A 10 YEAR DESCRIPTIVE STUDY OF CHILDREN AGED 0-12 YEARS REFERRED FOR EMERGENCY PSYCHIATRIC ASSESSMENT IN TALLAGHT UNIVERSITY HOSPITAL}

Erica Maguire, Caroline McGrath, Patricia Byrne*. Tallaght University Hospital, Dublin, Ireland

\subsection{6/archdischild-2019-epa.755}

Aims A review of the literature demonstrates that relatively little is known about acute psychiatric presentations in children (0-12 years). This study aims to review acute psychiatric presentations of children aged $0-12$ years to Tallaght University Hospital over a 10 year period.

Methods A retrospective descriptive case note study was completed. Subjects were defined as young people aged 12 years and under who were referred to the Psychiatric Liaison Service between July 2008 to June 2018. Data was anonymised and entered into a secure database for analysis.

Results 290 presentations of 241 children (0-12 years) were identified during the study period. $52 \%$ were male, $48 \%$ were female. The median age was 11 years. Children accounted for $7 \%$ of total referrals to the Psychiatric Liaison Service in 2008, however this figure had risen to $19 \%$ in 2018 . $30 \%$ more children were referred in 2017 compared to 2016.

Overall, the three most common primary presenting complaints were emotional dysregulation (25\%), conduct/oppositional defiant disorder (19\%) and anxiety disorder (17.5\%). 